\title{
Solving realistic portfolio optimization problems via metaheuristics: a survey and an example
}

\author{
Jana Doering ${ }^{1}$, Angel A. Juan² ${ }^{2}$ Renatas Kizys ${ }^{3}$, Angels Fito ${ }^{1}$, and Laura Calvet ${ }^{2}$ \\ ${ }^{1}$ Economics and Business Dept., Universitat Oberta de Catalunya, Barcelona, Spain \\ \{jdoering, afitob\} @uoc.edu \\ ${ }^{2}$ Computer Science Dept., Universitat Oberta de Catalunya - IN3, Castelldefels, Spain \\ \{ajuanp, lcalvet 1$\} @$ @uoc.edu \\ ${ }^{3}$ Subject Group Economics and Finance, University of Portsmouth, Portsmouth, UK \\ renatas.kizyseport.ac.uk
}

\begin{abstract}
Computational finance has become one of the emerging application fields of metaheuristic algorithms. In particular, these optimization methods are quickly becoming the solving approach alternative when dealing with realistic versions of financial problems, such as the popular portfolio optimization problem (POP). This paper reviews the scientific literature on the use of metaheuristics for solving rich versions of the POP and illustrates, with a numerical example, the capacity of these methods to provide high-quality solutions to complex POPs in short computing times, which might be a desirable property of solving methods that support real-time decision making.
\end{abstract}

Keywords: Metaheuristics, financial applications, portfolio optimization.

\section{Introduction}

Since the last century the direct relationship between financial decisions and wealth creation through capital accumulation or economic development has been widely accepted and thus been the center of much research in modern academia. Most of the questions in financial economics are formulated as optimization problems. Traditionally, exact methods have been employed in determining optimal solutions. This, however, requires modeling the problem subject to strict assumptions and simplifying formulations to render it solvable. Because this approach neglects depicting the intricacies of the real-life problems that decision-makers face in their everyday actions, the results are largely not transferrable to operations without reservations. Furthermore, the extraordinary internationalization and integration of financial markets and institutions has caused the decision-making process to become more complex. Advances in operations research and computer sciences have brought forward new solution approaches in optimization theory, such as metaheuristics [28]. While simple heuristics have been employed since the 1960s, the more advanced metaheuristics have only recently become relevant with the increase in computing power. They have shown to provide solutions to problems for which traditional 
methods are not applicable [22] in addition to providing near-optimal solutions to complex-combinatorial-optimization problems. Metaheuristics are conceptually simple, relatively easy to implement, and usually require little computational time, making them attractive for problem-solving in applications requiring real-time decisions. The application of metaheuristics to financial problems is summarized in Gilli et al. [9]. While metaheuristics do not guarantee finding a global optimal solution, Gilli and Schumann [10] point out that the goal of optimization in most reallife instances is not to provide an optimal solution, but one that fulfills the objectives to a satisfactory extent and hence promote the use of heuristic approaches. With respect to exact methods that provide an optimal solution to approximated problem formulations, a near-optimal solution to the unrestricted problem combined with reallife constraints might be preferable.

Since Markowitz [20] developed the modern portfolio theory centered on the mean-variance approach the academic community has been highly engaged in advancing the tools for portfolio optimization. The theory is based on two constituting assumptions, namely the financial investor being concerned with the expected returns and the risk of their respective investment. It is thus the goal to minimize the level of risk expressed through the portfolio variance for a given expected return level, resulting in the unconstrained efficient frontier. This established the portfolio optimization problem (POP), in which the risk is sought to be minimized based on a minimum expected return required by the investor. Fig. 1 shows the increasing popularity of metaheuristics for solving the POP. It becomes obvious that the trend in publications is increasing and that metaheuristics have received increased attention as solution approaches in the area of portfolio optimization.

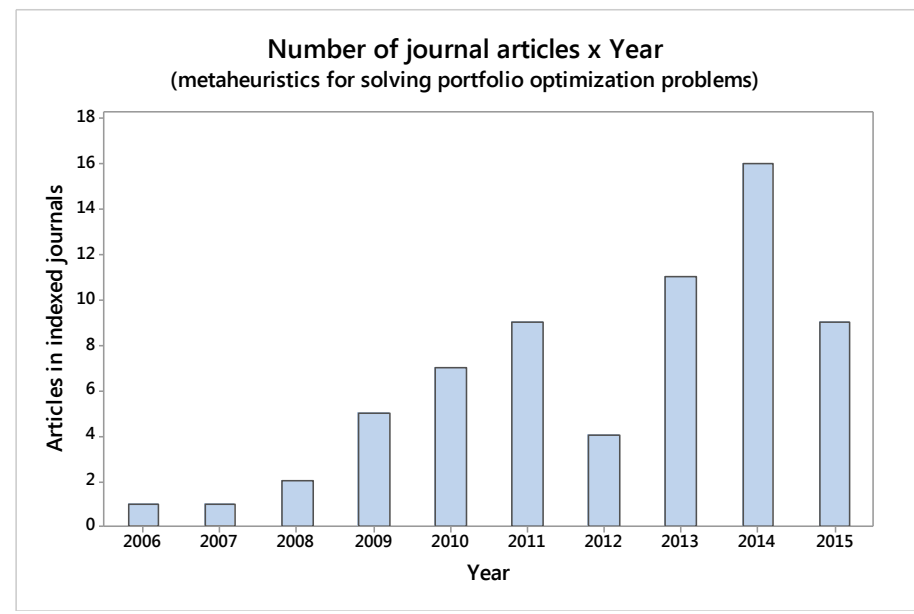

Fig. 1. Number of published articles per year on applications of metaheuristics in POP

While Mansini et al. [18] provide an extensive review on different portfolio optimization problems, including its historical evolution and the use of exact methods, our paper focuses on more recent contributions of metaheuristics for solving realistic 
versions of the POP, both including single-objective and multi-objective optimization. This paper also includes a computational example that illustrates the potential of metaheuristics in the field.

\section{The single-objective portfolio optimization problem}

While the original Markowitz problem can be solved using quadratic programming, metaheuristics have increasingly been employed to cope with the fact that the problem becomes NP-hard when real-life constraints are introduced [3]. These constraints include cardinality constraints (restricting the number of assets in the portfolio) and minimum proportional restrictions for inclusion of any asset. The classical version of the POP can be considered a single objective optimization problem with either one of the following model formulations: The investor minimizes the risk exposure subject to a minimum attainable expected return or the investor maximizes the expected return for a given level of risk. The classical POP can be formulated as follows [4]. The risk expressed as the portfolio variance is minimized:

$$
\sum_{i=1}^{N} \sum_{j=1}^{N} w_{i} * w_{j} * \sigma_{i j},
$$

subject to a minimum return, the constraint that the weights have to add up to one and the constraint that all asset weights must lie between zero and one, inclusive.

$$
\begin{aligned}
& \sum_{i=1}^{N} w_{i} * \mu_{i} \geq R^{*}, \\
& \sum_{i=1}^{N} w_{i}=1, \\
& 0 \leq w_{i} \leq 1 ; i=1, \ldots, N,
\end{aligned}
$$

where $N$ is the total number of assets available, $\mu_{i}$ is the expected return of an asset $i$, $R^{*}$ is the minimum required return, $w$ are the respective weights of the assets making up the portfolio and $\sigma_{i j}$ is the covariance between two assets $i$ and $j$.

Chang et al. [4] solve the above classical problem definition using three different metaheuristic approaches in order to generate a cardinality-constrained efficient frontier: genetic algorithm (GA), tabu search (TS), and simulated annealing (SA). They suggest pooling the results from the different approaches because no single heuristic was uniformly dominating in all observed datasets. Following this suggestion and combining GA, TS, and SA, Woodside-Oriakhi et al. [31] further explore the pooling option. They find that, on average, SA contributes little to the performance of the process and that thus a pooled GA and TS heuristic is superior to single metaheuristic approaches at the expense of higher computational time. By combining exact and metaheuristic methods, they create matheuristics. However, Soleimani et al. [26] introduce sector capitalization and minimum transaction lots as further constraints and found that the GA they developed outperformed TS, and SA. Particle swarm optimization (PSO) was found to be competitive with all three, GA, 
TS, and SA for the cardinality-constrained portfolio selection problem and especially successful in low-risk portfolios [5]. Golmakani and Fazel [11] further introduce minimum transaction lots, bounds on holdings and sector capitalization in addition to cardinality constraints and apply a combination of binary PSO and improved PSO that they call CBIPSO and found that, especially for large-scale problems, CBIPSO outperforms GA in that it provides better solutions in less computing time.

Di Tollo and Roli [8] provide a survey concerned with the early applications of metaheuristics to the POP and some of the proposed constraints. They explicitly highlight the potential use of hybrid approaches. Such a hybrid method was proposed by Maringer and Kellerer [19] who employ hybrid local search algorithm, which combines principles of SA and evolutionary algorithms (EA), to optimize a cardinality-constrained portfolio. The option of hybrid approaches is further investigated by Di Gaspero et al. [7] who combine a local search metaheuristics with quadratic programming to optimize a portfolio while accounting for cardinality constraints, lower and upper boundaries for the quantity of an included asset and preassignment constraints. According to their results, the developed solver finds the optimal solution in several instances and is at least comparable to other state of the art methods.

\section{The multi-objective portfolio optimization problem}

While single objective optimization methods consider either a minimal risk for a given return or a maximum risk for a given expected return or an objective function that weights the two goals and thus have to be run several times with the respective weights [23], multi-objective optimization methods find a set of Pareto solutions, while balancing two or more objective functions simultaneously. According to Streichert et al. [27] the problem can then be formulated as follows. For a multiobjective optimization it becomes necessary to minimize the portfolio risk expressed by the portfolio variance:

$$
\sum_{i=1}^{N} \sum_{j=1}^{N} w_{i}^{*} w_{j}^{*} \sigma_{i j}
$$

while maximizing the return of the portfolio

$$
\sum_{\mathrm{i}=1}^{\mathrm{N}} \mathrm{w}_{\mathrm{i}} * \mu_{\mathrm{i}}
$$

subject to

$$
\begin{aligned}
& \sum_{i=1}^{N} w_{i}=1 \\
& 0 \leq \mathrm{w}_{\mathrm{i}} \leq 1 ; \mathrm{i}=1, \ldots, \mathrm{N} .
\end{aligned}
$$


Zhu et al. [32] aim at a comparison of GA and particle swarm optimization (PSO) in solving the non-linear portfolio optimization problem with multi-objective functions. They argue that PSO overcomes the problem of increased convergence time in large instances expected for GA. They test their methodology on unconstrained, as well as constrained portfolios. While they do not include constraints other than a total weight equal to one, in addition to restricted portfolios, in which the short-selling of the portfolio's underlying assets is prohibited and thus all asset weights are positive, the authors also investigate unrestricted portfolios. However, they introduce the Sharpe ratio as a simultaneous measure of risk and return and thus turn the multi-objective optimization problem into a single objective optimization by optimizing an objective function that serves as a simultaneous measure of risk and return. The solution portfolios obtained with the PSO solver outperformed those constructed using GA for all test problems in terms of Sharpe ratio and the established efficient frontier was above that of GA portfolios in all but one instance. Enhanced PSO algorithms for solving the multi-objective POP have been proposed by Deng et al. [6] and He and Huang [12]. Cardinality and bounding constraints are incorporated by Deng et al. [6], who find that their algorithm mostly outperforms GA, SA, and TS algorithms as well as previous PSO approaches especially in the case of low-risk portfolios. It can be concluded that different findings unanimously favor PSO in situations when low-risk investment is demanded. Similarly, He and Huang [12] propose a modified PSO (MPSO) algorithm that outperforms regular PSO for their four different optimization sets. These sets consist of the traditional Markowitz mean-variance formulation and three alternative discontinuous objective functions that simultaneously account for minimizing risk while maximizing returns. More recently, they also developed a new PSO to further enhance discontinuous modeling of the POP and find that it generally outperforms PSO and also performs better than MPSO in larger search spaces [13]. Other population-based algorithms applied in optimizing portfolios include firefly algorithms (FA) [29] and artificial bee colony (ABC) algorithms. The authors developed these to address unconstrained portfolio optimization as well as portfolios with cardinality and bounding constraints. However, because the results were satisfactory at most even after modifications, the authors hybridized FA and ABC by incorporating the FA's search strategy into $\mathrm{ABC}$ to enhance exploitation and found that their data suggested superiority of the methodology compared to GA, SA, TS, and PSO [30] for unconstrained and cardinality-constrained portfolios.

Streichert et al. [27] account for further constraints, namely buy-in thresholds (acquisition prices) and roundlots (smallest volume of an asset that can be purchased). They treat the POP as a multi-objective optimization problem, in which they simultaneously minimize risk while maximizing expected returns through two multiobjective evolutionary algorithms (MOEA): GA and evolutionary algorithm (EA) enhanced through the integration of a local search that applies Lamarckism. They found that this enhancement greatly improved the reliability of the results, especially with respect to the additional constraints. However, there is a second point of criticism to the original Markowitz model, namely its assumption of normal financial returns, which, in reality are characterized by a leptokurtic and fat-tailed distribution [15], making it necessary to consider non-parametric risk measures. Such a measure is the value-at-risk as employed by Babaei et al. [2], who developed two multi-objective 
algorithms based on PSO to solve a cardinality- and quantity-constrained POP. Through splitting the whole swarm into sub-swarms that are then evolved distinctly their methodology outperformed similar benchmark metaheuristics. In order to optimize a non-parametric value-at-risk and to include further constraints, including a lower and upper bounds for the weights of included assets, a threshold for asset weight changes, lower and upper bounds for the weights of one asset class and a turnover rate that determines the maximum asset allocation changes possible at once, Krink \& Paterlini [16] developed the differential evolution for multi-objective portfolio optimization (DEMPO) algorithm, partly based on differential evolution (DE). An extended version of a generalized DE metaheuristic is also employed in optimizing a highly constrained POP by Ayodele and Charles [1]. The included constraints consist of bounds on holdings, cardinality, minimum transaction lots, and expert opinion. An expert can form an opinion based on indicators beyond the scope of the analyzed data and influence whether or not an asset should be included. Their methodology shows improved performance when compared to GA, TS, SA, and PSO. Lwin et al. [17] considered cardinality, quantity, pre-assignment and round lot constraints and developed a multi-objective evolutionary algorithm that is improved through a learning-guided solution generation strategy, which promotes efficient convergence (learning-guided multi-objective evolutionary algorithm with external archive, MODEwAwL). It was shown that the developed algorithm outperforms four benchmark state of the art multi-objective evolutionary algorithms in that its efficient frontier is superior. An extensive review of the application of evolutionary algorithms is provided by Metaxiotis and Liagkouras [21].

\section{A numerical example}

In Kizys et al. [14], the authors describe a metaheuristic algorithm that allows to solve a realistic version of the POP. In order to test the efficiency of their approach, we have used their algorithm with a set of data retrieved from the repository ORlib (http://people.brunel.ac.uk/ mastijb/jeb/orlib/portinfo.html). These instances were proposed by Chang et al. [4] and were studied by Schaerf [25], Moral-Escudero et al. [24], and Di Gaspero et al. [7]. The data set comprises constituents of five stock market indices, Hang Seng (Hong Kong), DAX 100 (Germany), FTSE 100 (United Kingdom), S\&P 100 (United States) and NIKKEI 225 (Japan). Following Di Gaspero et al. [7] we divided the portfolio frontier into 100 equidistant points on the vertical axis that represents the user-defined rate of portfolio expected return. The algorithm was executed on a constrained mean-variance frontier. The benchmark constraints are those imposed by the previous authors. Fig. 2 shows the values of average percentage loss (APL) and associated computational times for several algorithms. Notice that, for most instances, the algorithm from Kizys et al. [14] is able to outperform, in terms of the minimum APL (the lower the better), the algorithms proposed by Moral-Escudero et al. [24] and Di Gaspero et al. [7]. Also, the algorithm of the former authors shows its efficiency in terms of computational times requested to obtain a high-quality solution (Fig. 3). 


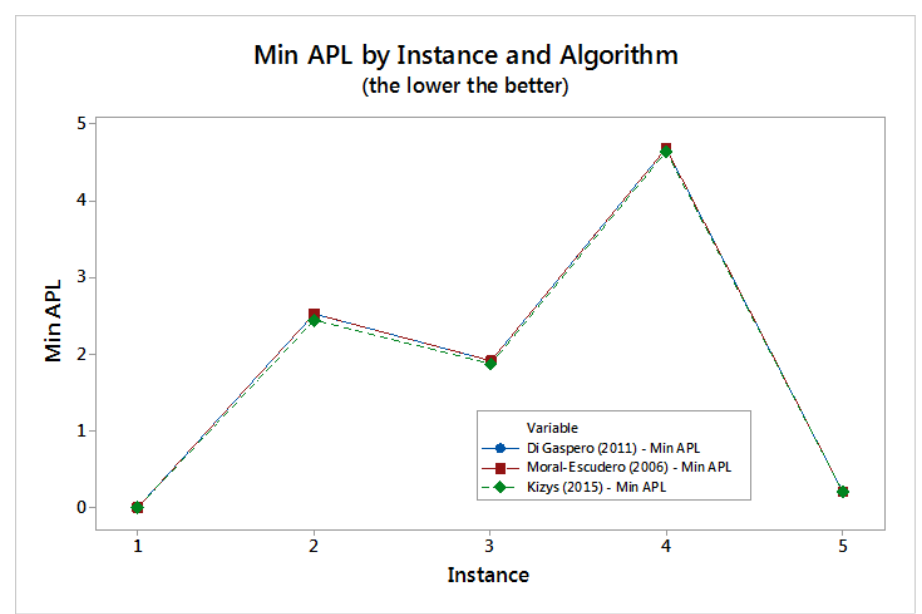

Fig. 2. Min APL (the lower the better) by instance and algorithm

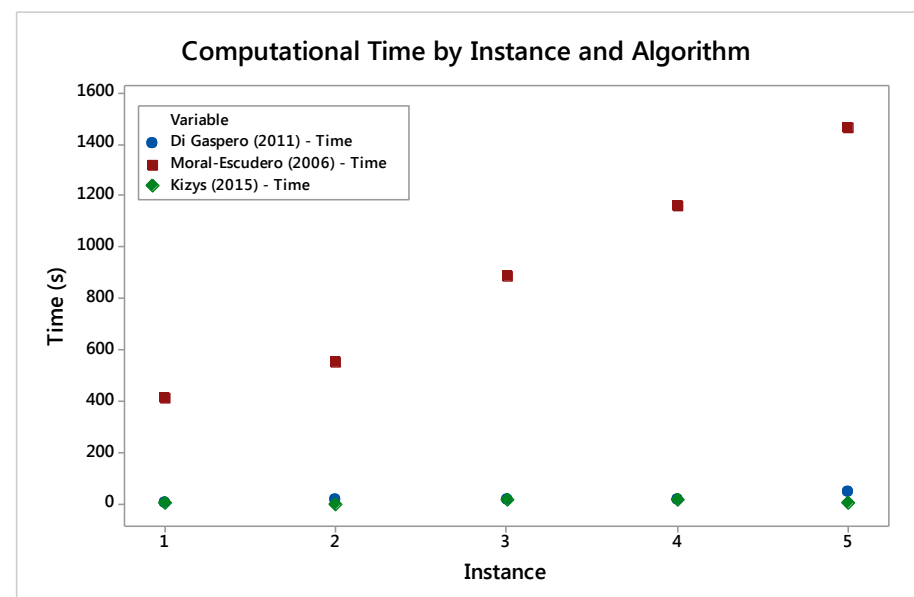

Fig. 3. Computational time by instance and algorithm

\section{Conclusions}

This paper analyzes the role of metaheuristic-based approaches in solving realistic variants of the well-known portfolio optimization problem, either with single or with multiple objectives. As discussed in the paper, metaheuristic algorithms are gaining popularity to solve these rich variants of the problem, since they might be used even in those scenarios in which exact methods cannot provide optimal solutions in reasonable computing times. An example of application illustrates the efficiency of 
these algorithms, in particular their capacity to provide high-quality solution in short computing times.

\section{Acknowledgments}

This work has been partially supported with doctoral grants from the UOC, the Spanish Ministry of Economy and Competitiveness (grants TRA2013-48180-C3-3-P, TRA2015-71883-REDT) and FEDER. Likewise we want to acknowledge the support received by the Department of Universities, Research \& Information Society of the Catalan Government (Grant 2014-CTP-00001).

\section{References}

1. Ayodele, A.A., Charles, K.A.: Portfolio Selection Problem Using Generalized Differential Evolution 3. 9, 42, 2069-2082 (2015).

2. Babaei, S., Sepehri, M.M., Babaei, E.: Multi-objective portfolio optimization considering the dependence structure of asset returns. Eur. J. Oper. Res. 244, 525-539 (2015).

3. Beasley, J.E.: Portfolio Optimisation : Models and Solution Approaches. In: Topaloglu, H. and Smith, J.C. (eds.) 2013 Tutorials in Operations Research: Theory Driven by Influential Applications. pp. 201-221. INFORMS (2013).

4. Chang, T.J., Meade, N., Beasley, J.E., Sharaiha, Y.M.: Heuristics for cardinality constrained portfolio optimisation. Comput. Oper. Res. 27, 1271-1302 (2000).

5. Cura, T.: Particle swarm optimization approach to portfolio optimization. Nonlinear Anal. Real World Appl. 10, 2396-2406 (2009).

6. Deng, G.-F., Lin, W.-T., Lo, C.-C.: Markowitz-based portfolio selection with cardinality constraints using improved particle swarm optimization. Expert Syst. Appl. 39, 45584566 (2012).

7. Di Gaspero, L., Di Tollo, G., Roli, A., Schaerf, A.: Hybrid metaheuristics for constrained portfolio selection problems. Quant. Financ. 11, 1473-1487 (2011).

8. Di Tollo, G., Roli, A.: Metaheuristics for the portfolio selection problem. Int. J. Oper. Res. 6, 13-35 (2008).

9. Gilli, M., Maringer, D., Schumann, E.: Numerical Methods and Optimization in Finance. Academic Press, Oxford (2011).

10. Gilli, M., Schumann, E.: Heuristic optimisation in financial modelling. Ann. Oper. Res. 193, 129-158 (2012).

11. Golmakani, H.R., Fazel, M.: Constrained portfolio selection using particle swarm optimization. Expert Syst. Appl. 38, 8327-8335 (2011).

12. He, G., Huang, N.: A modified particle swarm optimization algorithm with applications. Appl. Math. Comput. 219, 1053-1060 (2012).

13. He, G., Huang, N.: A new particle swarm optimization algorithm with an application. Appl. Math. Comput. 232, 521-528 (2014).

14. Kizys, R., Juan, A., Sawik, B. Linares, A.: Solving the Portfolio Optimization Problem under realistic constraints. In: Proceedings of the ICRA6/Risk 2015 Int. Conference, pp. 457-464 (2015).

15. Krink, T., Paterlini, S.: Differential evolution and combinatorial search for constrained index-tracking. Ann. Oper. Res. 172, 153-176 (2009). 
16. Krink, T., Paterlini, S.: Multiobjective Optimization Using Differential Evolution for RealWorld Portfolio Optimization. Comput. Manag. Sci. 8, 157-179 (2011).

17. Lwin, K., Qu, R., Kendall, G.: A learning-guided multi-objective evolutionary algorithm for constrained portfolio optimization. Appl. Soft Comput. 24, 757-772 (2014).

18. Mansini, R., Ogryczak, W., Speranza, M.G.: Twenty years of linear programming based portfolio optimization. Eur. J. Oper. Res. 234, 518-535 (2014).

19. Maringer, D., Kellerer, H.: Optimization of cardinality constrained portfolios with a hybrid local search algorithm. OR Spectr. 25, 481-495 (2003).

20. Markowitz, H.M.: Portfolio selection. J. Finance. 7, 77-91 (1952).

21. Metaxiotis, K., Liagkouras, K.: Multiobjective Evolutionary Algorithms for Portfolio Management: A comprehensive literature review. Expert Syst. Appl. 39, 11685-11698 (2012).

22. Michalewicz, Z., Fogel, D.B.: How to solve it : modern heuristics. Springer, Berlin (2004).

23. Mishra, S.K., Panda, G., Majhi, R.: Constrained portfolio asset selection using multiobjective bacteria foraging optimization. Oper. Res. 14, 113-145 (2014).

24. Moral-Escudero, R., Ruiz-Torrubiano, R., Suárez, A.: Selection of optimal investment portfolios with cardinality constraints. In: IEEE Congress on Evolutionary Computation. IEEE Press, pp. 2382-2388 (2006).

25. Schaerf, A.: Local search techniques for constrained portfolio selection problems. Computational Economics 20, 177-190 (2002).

26. Soleimani, H., Golmakani, H.R., Salimi, M.H.: Markowitz-based portfolio selection with minimum transaction lots, cardinality constraints and regarding sector capitalization using genetic algorithm. Expert Syst. Appl. 36, 5058-5063 (2009).

27. Streichert, F., Ulmer, H., Zell, A.: Evolutionary Algorithms and the Cardinality Constrained Portfolio Optimization Problem. In: Operations Research Proceedings 2003, Selected Papers of the International Conference on Operations Research (OR 2003). pp. 253-260 (2003).

28. Talbi, E.-G.: Metaheuristics: From Design to Implementation. John Wiley \& Sons, Hoboken, NJ (2009).

29. Tuba, M., Bacanin, N.: Artificial Bee Colony Algorithm Hybridized with Firefly Algorithm for Cardinality Constrained Mean-Variance Portfolio Selection Problem. Appl. Math. Inf. Sci. 8, 2831-2844 (2014).

30. Tuba, M., Bacanin, N.: Upgraded Firefly Algorithm for Portfolio Optimization Problem. In: 2014 UKSim-AMSS 16th International Conference on Computer Modelling and Simulation. pp. 113-118 (2014).

31. Woodside-Oriakhi, M., Lucas, C., Beasley, J.E.: Heuristic algorithms for the cardinality constrained efficient frontier. Eur. J. Oper. Res. 213, 538-550 (2011).

32. Zhu, H., Wang, Y., Wang, K., Chen, Y.: Particle Swarm Optimization (PSO) for the constrained portfolio optimization problem. Expert Syst. Appl. 38, 10161-10169 (2011). 